\title{
Gonadotropin-releasing hormone agonist and depot medroxyprogesterone acetate following uterine artery embolization in the management of uterine arteriovenous malformation
}

\author{
Dr. Padma Krishnaswamy, ${ }^{1}$ Dr. Indushekar Subbanna, ${ }^{1}$ Dr. Gayatri Devi \\ Sivasambu, ${ }^{1}$ Dr. Urvashi Thukral ${ }^{1}$
}

\begin{abstract}
Keywords: Arteriovenous malformation, uterine artery embolization, gonadotropin-releasing hormone agonist, depot medroxyprogesterone acetate
\end{abstract}

\begin{abstract}
A young woman presenting with recurrent episodes of uterine bleeding due to an extensive uterine arteriovenous malformation was treated with two sessions of uterine artery embolization. Further bleeding was managed with a combination of gonadotropin-releasing hormone and depot medroxyprogesterone acetate. The patient remains asymptomatic with a significant decrease in uterine and pelvic vascularity at one year follow-up.
\end{abstract}

These drugs may decrease the need for repeat embolization.

${ }^{1}$ M.S.Ramaiah Medical College, Bangalore, India

\section{Introduction}

Uterine arteriovenous malformation is rare but causes a serious, lifethreatening hemorrhage. Uterine artery embolization is the recommended treatment for recurrent episodes of bleeding in these cases. There is also a place for medical therapy as an adjunct to embolization to further decrease the vascularity. We present a case of extensive uterine arteriovenous malformation treated by transcatheter embolization followed by sequential gonadotropin-releasing hormone and depot medroxyprogesterone acetate.

\section{Case report}

A 29 year old G3P1021 presented to our emergency department on 29.10.12 complaining of sudden, excessive vaginal bleeding for 2 hours. Her recent history revealed the following: she had been treated in a local hospital $31 / 2$ weeks previously, with a diagnosis of missed abortion at 12 weeks of gestation, she had received tablets for termination of pregnancy (details not known). Not responding to this, she had undergone a dilatation and curettage on 05.10.12. During the procedure, she had received two units of blood transfusion due to excessive bleeding and a hemoglobin of $4.5 \mathrm{gm} \%$. There were

Please cite this paper as: Krishnaswamy P, Subbanna I, Sivasambu GD, Thukral U. Gonadotropin-releasing hormone agonist and depot medroxyprogesterone acetate following uterine artery embolization in the management of uterine arteriovenous malformation. Proc Obstet Gynecol. 2014;4(1): Article 4 [ 12 p.]. Available from: http://ir.uiowa.edu/pog/. Free full text article.

Corresponding author: Padma Krishnaswamy, M.S.Ramaiah Medical College, Bangalore, India. padmamsrmc@gmail.com

Financial Disclosure: The authors report no conflict of interest.

Copyright: (c) 2014 Krishnaswamy et al. This is an open-access article distributed under the terms of the Creative Commons Attribution License, which permits unrestricted use, distribution, and reproduction in any medium, provided the original author and source are credited. 
no reports of any histopathological examination. She had persistent vaginal bleeding intermittently resulting in a pelvic ultrasound on 18.10.12. This showed that the upper half of the uterus and the cervix were normal. The mid and lower corpus of the uterus was grossly enlarged, measuring $7.4 \times 6.5 \times 10.0 \mathrm{~cm}$, with a complex echotexture and increased vascularity with low resistance flow. (PI of 0.72-0.62 and RI of 0.52-0.50). The anterior wall of the uterus had a complex echotexture with multiple dilated blood vessels and multiple lakes. The impression was of an arteriovenous malformation following $D$ and $C$ or persistent trophoblastic tissue filling the cavity and penetrating the myometrium. With a serum $\beta$-HCG level of $122 \mathrm{mIU} / \mathrm{ml}$ on 19.10.12, she was prescribed methotrexate $5 \mathrm{mg}$ tablets for 5 days. She was seen by us on 29.10.12 with a sudden bout of vaginal bleeding.

Her obstetric history showed an uneventful lower segment caesarean section 6 years previously and a spontaneous abortion at 6 weeks about a year ago. There was no history of excessive bleeding in these two pregnancies.

Her previous menstrual cycles were normal with no history of excessive flow.

At admission, she was pale with a pulse of $112 / \mathrm{min}$, BP of $116 / 76 \mathrm{~mm}$ Hg. Physical examination was normal. There was moderate vaginal bleeding with a closed cervical os and a bulky uterus and no palpable adnexal mass. Her hemoglobin $(\mathrm{Hb})$ was $6 \mathrm{gm} \%$ and serum $\beta$-HCG level was $27 \mathrm{miu} / \mathrm{ml}$. The ultrasound scan showed a bulky uterus of $11.0 \times 4.5 \times 5.8 \mathrm{~cm}$ with fundus and cervix appearing normal.
Figures 1-4 - Angiography during the first session of embolization showing the rapid drainage of the dye before and a significant decrease in flow following embolization (figures 1 and 2- right pelvic vessels; figures 3 and 4- left pelvic vessels)

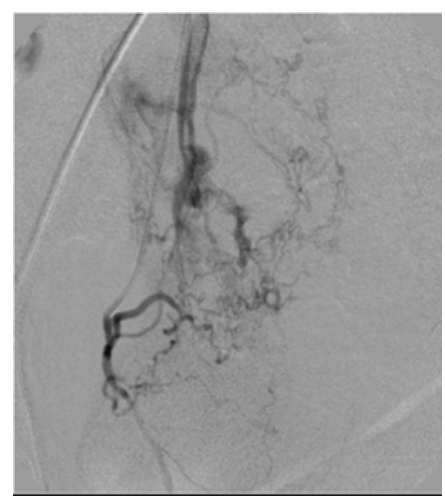

Figure 1

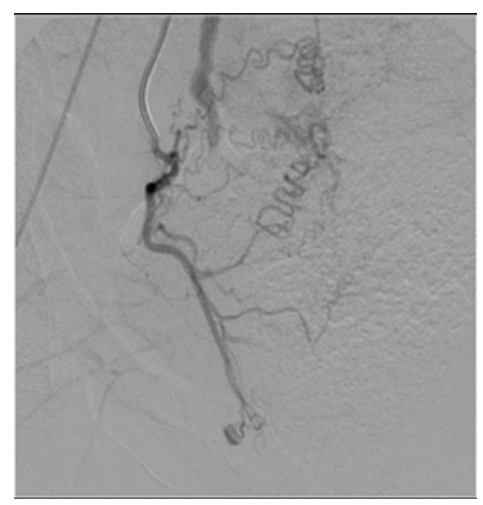

Figure 2

A mixed echogenic, vascular lesion in the region of the right adnexa and the right lateral wall of the uterus measuring $5.0 \times 5.0 \mathrm{~cm}$ was noted. Both ovaries were normal. MRI suggested a uterine arteriovenous malformation (AVM) with a heterogenous area in the body of the uterus measuring $9.4 \times 4.1 \times 3.5 \mathrm{~cm}$ with multiple circumferential flow voids with marked enhancement on contrast. The 
fundus of the uterus and cervical region appeared normal with normal endometrium thickness. She was transfused 2 units of packed red cells and managed with uterine artery embolization on 30.10.12. The initial internal iliac angiograms showed extensive pelvic $\mathrm{A} \vee \mathrm{m}$ malformation with hypervascularity of the uterine wall and early filling of draining veins. The feeding arterial branches of uterine walls were occluded with polyvinyl alcohol (PVA) particles 700 microns (Figures 1-4). The patient was stable with no further fresh bleeding and was discharged the next day.

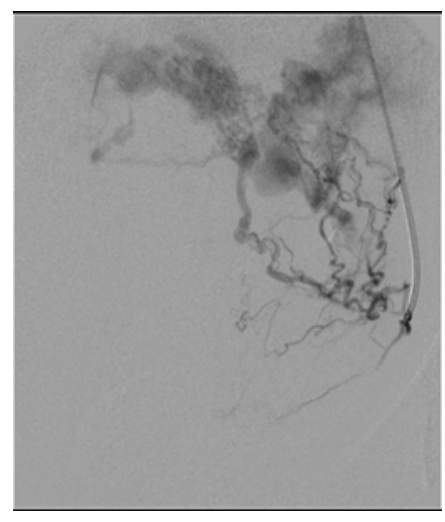

Figure 3

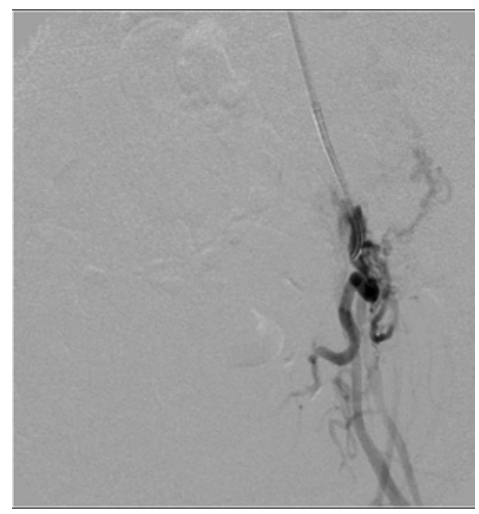

Figure 4

Uterine arteriovenous malformation
She presented again 13 days later on 11.11.2012 with a bout of excessive vaginal bleeding. She was pale with a pulse of $124 \mathrm{bpm}$, BP of 130/80 $\mathrm{mmHg}$ and moderate amount of bleeding. Her $\mathrm{Hb}$ was $9.8 \mathrm{gm} \%$ and serum $\beta$-HCG was not detected. Ultrasound showed residual $A V M$. Due to the extensive nature of the malformation seen during embolization, a CT angiogram was done which showed extensive pelvic AVM with feeders from multiple internal iliac branches and ovarian arteries. (Figure 5) Demonstration of exact feeder arterial anatomy was suboptimal because of multiple dilated and early filling of draining veins. The bleeding reduced and she was managed conservatively with tranexamic acid.

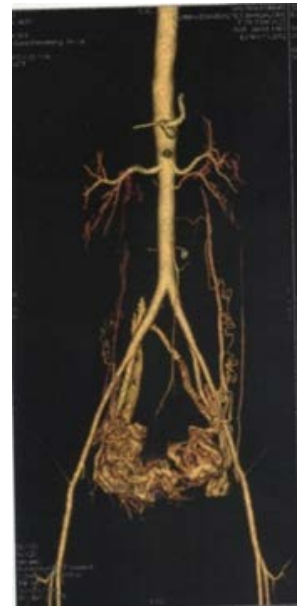

\section{Figure 5 - CT angiogram showing} extensive pelvic AVM

The patient was admitted again 3 weeks later on 07.12.12 with excessive bleeding and giddiness. The patient was anxious and pale, with a pulse of $88 / \mathrm{min}$, BP of $110 / 70 \mathrm{mmHg}$ and minimal bleeding. Hemoglobin $(\mathrm{Hb})$ was 11 gm\% and serum betaHCG was not detected. On 14.12.12, she underwent repeat embolization of uterine arteries with PVA particles 
followed by pelvic arterial embolization with cyanoacrylate glue (2cc of cyanoacrylate glue, Cook) mixed with Lipiodol Ultra-Fluide (Guerbet, France). The arteriovenous shunt on the right side of pelvis was also embolized similarly. Post-embolization angiography showed a significant reduction of flow in the pelvic vessels. (Figures 6,7) The patient was discharged after a day of observation.

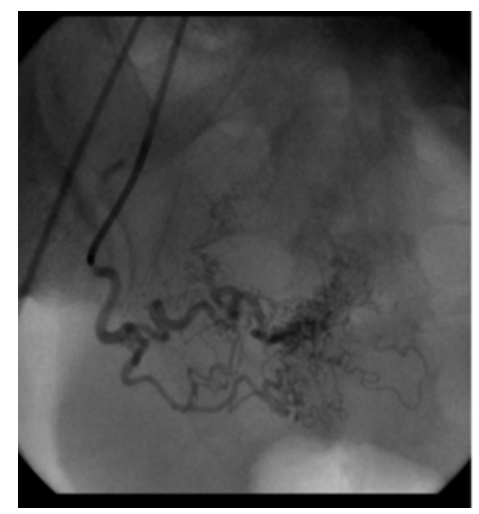

Figure 6 - Angiography during the second session of uterine artery embolization. Right pelvic vessels pre-embolization

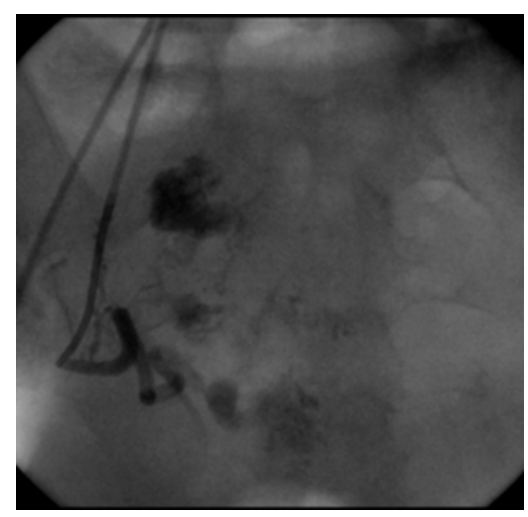

Figure 7- Post embolization showing significant obliteration
Four days later, on 18.12.12, the patient again had excessive vaginal bleeding and giddiness. She was immediately treated in a nearby hospital with intravenous fluids and later readmitted in our hospital. On examination she was pale, pulse of $80 / \mathrm{min}$, BP of $110 / 70 \mathrm{mmHg}$, with minimal bleeding and $\mathrm{Hb}$ of $9.5 \mathrm{gm} \%$. Ultrasound examination showed a heterogeneous area of $3.5 \times 2.0 \mathrm{~cm}$ with significant internal vascularity in the anterior wall of the uterus. There was another similar vascular lesion in the right adnexal region of $4.5 \times 2.5 \mathrm{~cm}$ abutting the uterus with the left adnexa also showing significant vascularity. The impression was a decreased but persistent bilateral pelvic and uterine arteriovenous malformation. (Figure 8) With bleeding reduced but still persistent, the patient and her relatives were very apprehensive and insistent on definitive management with hysterectomy. The consequences of hysterectomy in the context of extensive uterine arteriovenous malformation were discussed with them. A trial of medical management was agreed upon. She received gonadotropin-releasing hormone agonist (GnRHa) (Leuprolide depot) $3.75 \mathrm{mg}$ intramuscularly on 22.12 .12 with the premise that the vascularity seen near the endometrium would decrease and the endometrium would become thin preventing subsequent sloughing and bleeding. She was discharged with advice for a second dose the next month. 


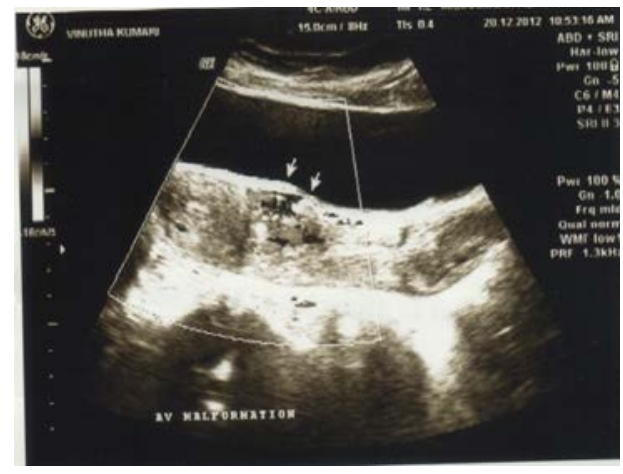

Figure 8 - Longitudinal view of uterus with mid and lower anterior wall showing AVM after two sessions of pelvic arterial embolization

Seen a month later, on 21.01.13, she was asymptomatic and the ultrasound scan showed a very significant decrease in vascularity both in the uterine myometrium and adnexal regions. With the side-effects and cost of $\mathrm{GnRHa}$ therapy being high, we started her on depot medroxyprogesterone acetate (DMPA) $150 \mathrm{mg}$ intramuscular.

An ultrasound two months later, on 25 .03.13 showed the uterus with normal uterine and pelvic vascularity. (Figures $9,10)$. We have continued with injections every three months of DMPA for a year which has also doubled as a reliable contraceptive. The patient has remained asymptomatic on regular follow-up and a favorable outcome with future pregnancies has been discussed with her.

\section{Discussion}

An arteriovenous malformation (AVM) is a tangle of blood vessels where arteries connect directly to veins without an intervening capillary network. It is a system of multiple feeding arteries, the tangle or nidus, and enlarged draining veins, forming a high pressure shunt or fistula. The vessels which become weak in this process can rupture and bleed.

Figures 9 and 10 - Uterus and right adnexa appear normal with normal vascularity.

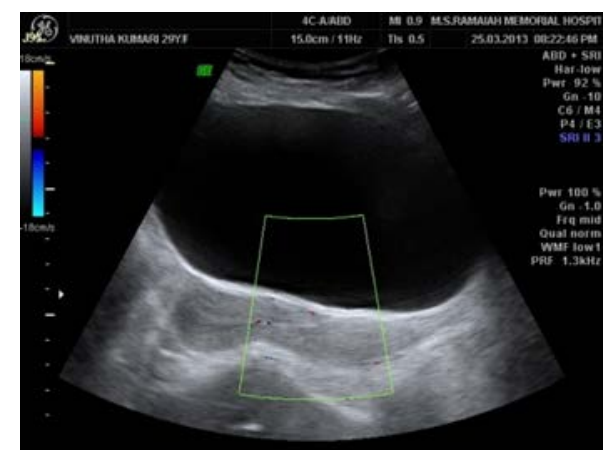

Figure 9

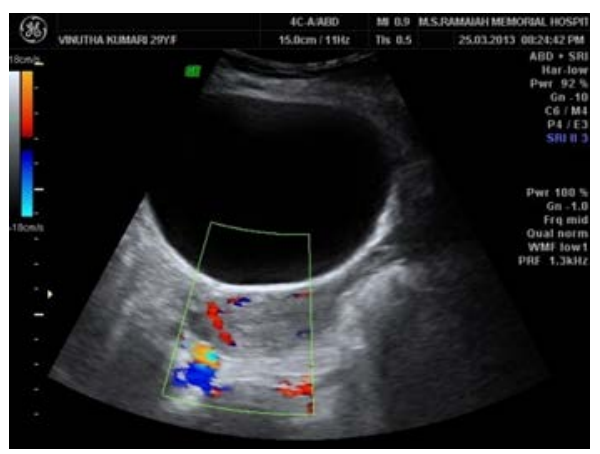

Figure 10

Uterine arteriovenous malformations result from the formation of multiple arterio-venous fistulous communications within the uterus. They can be congenital or acquired. Congenital AVMs are usually isolated anomalies and arise as a result of a defect in embryonic vascular differentiation or arrest of development. They may extend beyond the uterus and can grow as pregnancy progresses. Acquired AVMs, on the other hand, are usually 
secondary to previous uterine surgery, curettage, carcinoma of the cervix and endometrium or trophoblastic disease. Pregnancy is thought to play an important role in their pathogenesis. It is postulated that these malformations may arise when venous sinuses become incorporated in scars within the myometrium after necrosis of the chorionic villi. This may also explain the association of AVM with trophoblastic disease following chemotherapy. ${ }^{1} \quad$ Uterine trauma appears to be a common etiological factor in all these conditions with the potential for development of abnormal fistulous communication between arteries and veins in the uterine wall. Some of these communications may develop and persist as an AVM. $^{2}$

\section{Clinical features}

The clinical picture of uterine AVM can range from being asymptomatic to lifethreatening vaginal bleeding. The classical bleeding pattern is intermittent and torrential, suggesting an arterial hemorrhage. The vaginal bleeding can be life threatening in 30\% of patients requiring transfusion. The bleeding presumably occurs when the vessels become exposed as they erode or abut the endometrium or when the endometrium sloughs during menstruation. $^{2}$ They can also manifest as torrential bleeding following uterine curettage as there is often only a thin layer of endometrium overlying the malformation, which may be disrupted during the procedure. ${ }^{1}$ The time interval when the bleeding occurs after the inciting event also appears to be variable. ${ }^{3} \mathrm{~A}$ history of dilatation and curettage and/or uterine surgery has been reported 1 month to 7 years before the diagnosis with a median of 19 months. Any patient with unexplained vaginal bleeding with a history of therapeutic dilatation and curettage, abortion, previous uterine surgery, endometrial carcinoma or gestational trophoblastic disease should be suspected of uterine AVM regardless of how long back such events may have occurred. ${ }^{4}$

\section{Diagnosis}

Uterine AVM is usually first diagnosed with gray-scale ultrasonography and confirmed with color Doppler. While radiographic angiography remains the gold standard, MRI and CT angiography are non-invasive modalities which can provide additional information to help in management.

The gray-scale sonographic appearance of AVM can be variable and non-specific. The most common finding is the presence of multiple tubular anechoic spaces within the myometrium, without any mass effect. It gives a 'spongy' myometrial echo texture. Other sonographic features can be in the form of a subtle heterogeneous myometrium, a focal intramural mass mimicking a myoma or an endometrial mass resembling an endometrial polyp. ${ }^{5}$ Gynecologic vascular abnormalities should be considered in women of reproductive age with unexplained vaginal bleeding and in postmenopausal women in whom anechoic structures are detected on ultrasound. ${ }^{6}$ Further delineation of AVM is provided by color Doppler imaging which shows a tangle of vessels with multidirectional highvelocity flow that produces a color mosaic pattern. ${ }^{5}$ Duplex Doppler further reveals the classic features of arteriovenous shunting - a picture of a fast arterial flow with low resistance: high peak systolic velocity, an arterial spectral waveform with a high diastolic 
component, and a pulsatile highvelocity venous waveform with little variation in systolic-diastolic velocities. ${ }^{2,6}$

The differential diagnosis of a uterine AVM during grey-scale sonography includes multilocular ovarian cysts, hydrosalpinx, pelvic varicosities, gestational trophoblastic disease (GTD) and retained products of conception. On color Doppler ultrasonography, few or no vascular signals can be detected either in ovarian cysts or hydrosalpinx and pelvic varicosities can be differentiated by the depiction of prominent parametrial vessels with normal venous spectral waveforms. But retained products of conception and GTD appear as an endometrial- based mass that show uterine Doppler findings similar to an AVM. $^{7}$ Here a serum beta-HCG assay is required and the main differentiating point is positive $\beta$-HCG values. However, it should also be kept in mind that uterine AVM can coexist with GTD and retained products of conception. 6,7 Angiography is considered the reference standard for the definitive diagnosis of AVM. The classic angiographic features consist of a complex tangle of vessels supplied by enlarged feeding arteries, in association with early venous drainage during the arterial phase. ${ }^{2}$ It further helps to define the vascular anatomy, assess the extent of the vascular malformation, and identify the feeding vessels. But being an invasive procedure this is reserved for patients in whom surgical intervention or therapeutic transcatheter embolization is contemplated. A CT angiography may be a good substitute for pelvic angiography. A 3D-CT angiography is useful for making a differential diagnosis and for preoperative planning in patients with postabortion uterine hemorrhage. ${ }^{8}$

MR angiography also allows us to confirm the diagnosis of uterine AVM non-invasively. It is helpful in determining the magnitude of the vascular malformation, particularly if extrauterine extension is suspected. Moreover, additional pelvic disorders may be identified and better characterized with MRI. Serpiginous flow-related signal voids corresponding to the myometrial hypervascular areas on color Doppler sonography are characteristic of uterine AVMs. ${ }^{9}$

In our patient, excessive bleeding was noticed during the dilatation and evacuation procedure necessitating blood transfusion. She continued to bleed intermittently which led to further investigation with ultrasound revealing a uterine AVM. With a serum betaHCG of $122 \mathrm{mIU} / \mathrm{ml}$, she had received methotrexate tablets for ten days before coming to us. This is not a recommended management option. We could find one article expressing the usefulness of methotrexate in cases of AVM coexisting with retained chorionic villi. The authors recommended methotrexate therapy or a retrial of uterine curettage immediately after embolization to decrease recanalization of AVM that may occur due to rapid recruitment of collateral vessels from pelvic arteries in these situations. ${ }^{2}$ We did both an MRI and CT angiography in our patient. She had a low serum betaHCG level which could have been due to the presence of retained products of conception or more likely an occurrence in the normal course of a declining beta-HCG level. An MRI angiogram was done to confirm AVM, especially as it was seen extending to 
the parametrium and to rule out bleeding due to coexistent retained products of conception. A CT angiography was done after the first session of uterine arterial embolization when the patient presented with a sudden bout of bleeding. The intention was to identify the feeder vessels which would help in subsequent planning but because of the extensive nature of the malformation and early venous drainage, no specific feeder could be identified in the tangle of vessels. We found that the uterine AVM extended and appeared to involve the pelvic vasculature tempting us to make a diagnosis of a pelvic AVM as well.

\section{Management}

Following a definitive diagnosis of a uterine AVM, further management depends on the patient's hemodynamic status, amount of bleeding, age, and desire for future fertility. Acute management calls for stabilizing the hemodynamic status and controlling active bleeding. Intrauterine tamponade with a Foley's bulb can be a useful temporary measure to decrease bleeding. Treatment of acquired AVMs requires occlusion or resection of all arteriovenous communications to prevent further life-threatening hemorrhage.

Treatment options are expectant management, intra-arterial embolization or hysterectomy. Patients who are anemic or hemodynamically unstable are considered for angiography and embolization. This is the primary choice when interventional radiological expertise is available as it is safe and efficacious with fertility preservation. Patients with a single episode of bleeding who are hemodynamically stable can be observed, as many of them will remain asymptomatic and also because traumatic AVMs are reported to regress over time. ${ }^{10}$ If they have recurrent bleeding on follow-up, embolization is indicated. The decision for observation or embolization depends on the patient's clinical status rather than on the size of the AVM on imaging. ${ }^{6}$

Hysterectomy is reserved for patients with massive uterine hemorrhage, persistent evidence of AVM with intractable bleeding, or poor compliance with regular follow-up; however, surgery may be hazardous in the presence of extensive AVM with pelvic extension. A cautious approach with adequate preparation to deal with intra-operative hemorrhage is necessary and a more radical procedure may be required. ${ }^{11}$

Treatment with Transcatheter Arterial Embolization

Percutaneous transcatheter embolization is now an accepted, safe and highly effective technique for controlling obstetric and gynecologic hemorrhage. The uterine artery and the feeding arteries can be embolized with micro particles, coils, glue or alcohol, polyvinyl alcohol (PVA), cyanoacrylate glue, and stainless steel coils while uterine perfusion is maintained through collateral supply. ${ }^{3}$

The selection of embolic material depends on the size and range of the AVMs. The number of embolization procedures required also varies. A single embolization procedure may be sufficient for localized AVMs whereas multiple procedures are required in extensive, complex AVMs. Serial embolizations prevent exceeding of the contrast-material limits that can 
happen with lengthy angiographic procedures and also reduce the risk of excessive embolization, which could lead to tissue necrosis or complications resulting from postthrombosis edema. ${ }^{12}$ The clinical success rate after one to two embolization procedures is $93-96 \%$, and its complication rate is $4 \% .^{3}$ Most of the adverse events after uterine artery transcatheter embolization for AVM or obstetric hemorrhage are often minor when performed by expert interventional radiologists. Patients may experience pelvic pain and nausea for 12-24 hours after the procedure, which gradually decreases in the next 5-7 days. Major complications are rare and include uterine necrosis, sepsis, and lethal pulmonary embolism. ${ }^{9}$

Subsequent pregnancies following uterine artery embolization for AVM and postpartum hemorrhage are reported. Normal intrauterine pregnancies occurring after embolization with various embolic materials like Gelfoam, PVA particles and glue prove that an adequate collateral blood supply can develop to support a full-term pregnancy. There are also no reports of major complications of pregnancy and labor such as severe growth restriction or abnormal invasion of the placental bed in these patients. $^{13}$ Thus the advantages of uterine artery embolization include very high success rates, low complication rates, avoidance of surgical risks, and preservation of fertility with no further significant risks in subsequent pregnancies.

In our case the extensive nature of the malformation prompted us to plan it as a multistage procedure with the use of glue in the second session.
Medical management

Pharmacological treatment of uterine AVMs can be used for patients with minimal symptoms and good compliance. Estrogens and progestins, methylergonovine, danazol, 15-methylprostaglandin F2alpha, oral contraceptives and gonadotropinreleasing hormone agonist have been used though their role is not clear. There are no uniform guidelines and studies on which to base rational treatment in these rare and complex cases.

The mechanism of the beneficial effects of drugs on AVMs is not well understood. When methylergonovine maleate and estrogens were used successfully in a patient, it was hypothesized that methylergonovine maleate induced tetanic myometrial contractions to collapse the AVM and reduce its blood flow and intravenous conjugated estrogens helped by covering the hemorrhaging vessels with proliferative endometrium. ${ }^{14}$

The effect of gonadotropin-releasing hormone $(\mathrm{GnRH})$ agonists on uterine arteriovenous malformations has not been established but holds promise in their successful management. It has been used alone for six months with complete disappearance of the $\mathrm{AVM}^{15}$ or in conjunction with uterine artery embolization, where it was used over six months to decrease the size of the AVM with follow-up embolization to cause its complete disappearance. ${ }^{16}$

In our patient, arterial embolization was attempted to obliterate the feeding vessels of the AVM. When she presented with a sudden bout of bleeding four days after the second session of embolization, GnRH agonist was given because of its value in decreasing uterine vascularity in both 
myomatous and nonmyomatous tissues. We also envisaged that with the AVM being so close to the endometrium, the drug-induced endometrial changes would prevent shedding of the endometrium and exposing the AVM when given for a few months. Though planned as a multiple dose treatment, a single dose produced sufficient decrease in the vascularity of the uterus and pelvis, prompting us to follow the $\mathrm{GnRH}$ agonist therapy with depot medroxyprogesterone acetate (DMPA) to sustain the avascular status and prevent shedding of the endometrium. With our patient remaining asymptomatic with no side-effects at nine months follow-up, GnRH agonist therapy followed by DMPA may have the potential to be a conservative treatment modality for uterine arteriovenous malformations in hemodynamically stable patients with close follow-up.

\section{Conclusion}

To conclude, our case illustrates an acquired uterine AVM. Sonography with color Doppler is the primary investigation for screening and diagnosis. CT angiography or MRI angiography can be used to further delineate the vascular anatomy and rule out pelvic pathology in patients with extensive AVMs. They can be safely managed with selective embolization. Decreased but persistent, symptomatic AVMs postembolization can be effectively treated with GnRH agonist to further decrease their vascularity. Combining it with DMPA may be an effective, feasible, safe, and affordable method in the management of complex, lifethreatening uterine AVMs. The successful obliteration of the abnormal vascular channels in our case gives us hope that a single dose of $\mathrm{GnRH}$ agonist can be used along with long term depot medroxyprogesterone acetate following uterine artery embolization in those women who present with repeated bouts of sudden vaginal bleeding. These drugs may decrease the need for repeat embolization. We speculate that in places with no facilities for interventional radiological expertise, treatment with GnRH agonist and DMPA to decrease uterine vascularity may make a significant difference in the management of these difficult cases.

\section{References}

1. Kelly SM, Belli AM, Campbell S. Arteriovenous malformation of the uterus associated with secondary postpartum hemorrhage. Ultrasound Obstet Gynecol. 2003 Jun;21(6):6025. PubMed PMID: 12808679. http://dx.doi.org/10.1002/uog.148

2. Kwon JH, Kim GS. Obstetric iatrogenic arterial injuries of the uterus: diagnosis with US and treatment with transcatheter arterial embolization. Radiographics. 2002 JanFeb;22(1):35-46. PubMed PMID: 11796896.

http://dx.doi.org/10.1148/radiographics .22.1.g02ja0735

3. Ghai S, Rajan DK, Asch MR, Muradali D, Simons ME, TerBrugge KG. Efficacy of embolization in traumatic uterine vascular malformations. J VascIntervRadiol. 2003 Nov;14(11):1401-8. PubMed PMID: 14605105.

http://dx.doi.org/10.1097/01.RVI.00000 96761.74047.7D 
4. Lin AC, Hung YC, Huang LC, Chiu TH, Ho M. Successful treatment of uterine arteriovenous malformation with percutaneous embolization. Taiwan J Obstet Gynecol. 2007 Mar;46(1):60-3. PubMed PMID: 17389192. http://dx.doi.org/10.1016/S10284559(08)60109-6

5. O'Brien P, Neyastani A, Buckley AR, Chang SD, Legiehn GM. Uterine arteriovenous malformations: from diagnosis to treatment. J Ultrasound Med. 2006 Nov;25(11):1387-92; quiz 1394-5. PubMed PMID: 17060424.

6. Polat P, Suma S, Kantarcý M, Alper F, Levent A. Color Doppler US in the evaluation of uterine vascular abnormalities. Radiographics. 2002 Jan-Feb;22(1):47-53. PubMed PMID: 11796897.

7. Öner AY, Pocan S. Uterine Arteriovenous Malformation: GrayScale and Doppler sonography findings with CT angiography correlation. Gazi Medical Journal 2005;16(2)95-97.

8. Umezu T, Iwase A, Ota T, Suzuki K, Nakagawa A, Nakahara T, Takikawa S, Kobayashi H, Manabe S, Suzuki K, Goto M, Kikkawa F. Threedimensional CT angiography is useful for diagnosis of postabortion uterine hemorrhage: 3 case reports and review of the literature. J Minim Invasive Gynecol. 2010 MarApr;17(2):246-51. doi: 10.1016/j.jmig.2009.11.012. PubMed PMID: 20226418.

9. Maldonado J, Perez C, Rodriguez W. AJR Teaching File: Profuse vaginal bleeding seven weeks following induced abortion. AJR Am J Roentgenol. 2008 Dec;191(6 Suppl):S79-82. doi: 10.2214/AJR.07.7044. PubMed PMID: 19018054.
10. Timmerman D, Van den Bosch $T$, Peeraer K, Debrouwere E, Van Schoubroeck D, Stockx L, Spitz B. Vascular malformations in the uterus: ultrasonographic diagnosis and conservative management. Eur J ObstetGynecolReprod Biol. 2000 Sep;92(1):171-8. PubMed PMID: 10986453.

http://dx.doi.org/10.1016/S03012115(00)00443-7

11. Moulder JK, Garrett LA, Salazar GM, Goodman A. The role of radical surgery in the management of acquired uterine arteriovenous malformation. Case Rep Oncol. 2013 Jun 1;6(2):303-10. doi: 10.1159/000351609. PubMed PMID: 23898273; PubMed Central PMCID: PMC3725026.

12. Do YS, Yakes WF, Shin SW, Lee BB, Kim DI, Liu WC, Shin BS, Kim DK, Choo SW, Choo IW. Ethanol embolization of arteriovenous malformations: interim results. Radiology. 2005 May;235(2):674-82. PubMed PMID: 15858106. http://dx.doi.org/10.1148/radiol.235204 0449

13. Eling R, Kent A, Robertson $M$. Pregnancy after uterine arterio-venous malformation-case series and literature review. AJUM Australasian Journal of Ultrasound in Medicine. 2012 Aug;15(3):87-96.

14. Montanari L, Alfei A. P35.02: arteriovenous malformation of the uterus: successful pregnancy after medical treatment. Ultrasound Obstet Gynecol. $2007 \quad$ Oct;30(4):585. http://dx.doi.org/10.1002/uog.4836

15. Nonaka T, Yahata T, Kashima K, Tanaka K. Resolution of uterine arteriovenous malformation and successful pregnancy after treatment with a gonadotropin-releasing hormone agonist. Obstet Gynecol. 2011 Feb;117(2 Pt 2):452-5. doi: 10.1097/AOG.0b013e3181f7381f. PubMed PMID: 21252786. 
16. Morikawa $M$, Yamada $T$, Yamada $H$, Minakami $H$. Effect of gonadotropinreleasing hormone agonist on a uterine arteriovenous malformation.
Obstet Gynecol. 2006 Sep;108(3 Pt

2):751-3. PubMed PMID: 17018490. http://dx.doi.org/10.1097/01.AOG.0000 191584.28717.2c 